\title{
Modeling Microdefects Closure Effect with Isotropic/Anisotropic Damage
}

\author{
R. DESMORAT* \\ LMT-Cachan, ENS-Cachan/Université Paris 6/CNRS \\ 61, av. du président Wilson, F-94235 Cachan Cedex, France \\ S. CANTOURNET \\ Ecole des Mines de Paris, Centre des Matériaux Pierre-Marie \\ UMR CNRS 7633, BP 87, 91003 Evry Cedex, France
}

\begin{abstract}
Continuum damage mechanics (CDM) for metals is often written in terms of an isotropic (scalar) damage. In this case, solutions have been proposed to represent the differences of behavior in tension and in compression also called quasi-unilateral (QU) conditions or microdefects closure effect.

A recent anisotropic damage model has been developed to take into account the damage orthotropy induced by plasticity (Lemaitre, J., Demorat R. and Sauzay, M. (2000). Anisotropic Damage Law of Evolution, Eur. J. Mech. A/Solids, 19: 513-524). The purposes here are then two. First, a unified framework for isotropic and anisotropic damage is proposed. Then, it is to extend Ladevèze and Lemaitre's framework (Ladevèze, P. and Lemaitre, J. (1984). Damage Effective Stress in Quasi Unilateral Conditions, In: Proceedings of the 16th International Congress of Theoretical and Applied Mechanics, Lyngby, Denmark) for the QU conditions to anisotropic damage induced by plasticity.

Yield surfaces and damage versus accumulated plastic strain curves, drawn for different loading, illustrate the effect of the QU conditions on the damage evolution.
\end{abstract}

KEY WORDS: damage, anisotropy, unilateral effect, yield surfaces.

\section{INTRODUCTION}

\begin{abstract}
CONTINUUM DAMAGE MECHANICS (CDM) is a continuous representation of the initiation, growth, and coalescence of microdefects and/or micro-cracks present at a scale smaller than the scale of the representative
\end{abstract} *Author to whom correspondence should be addressed. E-mail: desmorat@1mt.ens-cachan.fr
Figure 2 appears in color online: http://ijd.sagepub.com

International Journal of DAMAge Mechanics, Vol. 17-January 2008 
volume element of classical continuum mechanics. Built within a thermodynamics framework, CDM uses as many internal variables as there are mechanisms of deformation and material degradation. It allows the behavior of materials up to crack initiation to be modeled.

The simplest representation of damage is a scalar representation (variable $D$ ), which corresponds to isotropy (Kachanov, 1958; Rabotnov, 1968; Lemaitre, 1971). The general damage anisotropy is represented by a fourth-order tensor (variable $\underline{\boldsymbol{D}}$; Chaboche, 1978; Leckie and Onat, 1981; Krajcinovic, 1985), but for practical applications a second-order damage tensor is often used (Cordebois and Sidoroff, 1982; Ladevèze, 1983; Chow and Wang, 1987; Murakami, 1988; Lemaitre et al., 2000; Lemaitre and Demorat, 2001, 2005). This representation is considered here and the corresponding damage tensor will be denoted as $\boldsymbol{D}$.

Damage modifies the elastic properties of a material and an effective stiffness fourth-order tensor $\underline{\underline{E}}$ is commonly introduced as:

$$
\boldsymbol{\sigma}=\underline{\tilde{E}}: \boldsymbol{\epsilon}^{e}
$$

where $\epsilon^{e}$ is the elastic strain and $\underline{\tilde{E}}=\underline{\boldsymbol{M}}^{-1}: \underline{\boldsymbol{E}}$ are, respectively, the initial and the effective Hooke's tensors for anisotropic damage represented in the isotropic case by Young's modulus $E$ and the effective Young's modulus $\tilde{E}=E(1-D)$. The fourth-order tensor $\underline{\boldsymbol{M}}$ also defines the effective stress $\tilde{\boldsymbol{\sigma}}$ such as:

$$
\tilde{\boldsymbol{\sigma}}=\underline{\boldsymbol{M}}: \boldsymbol{\sigma}=\underline{\boldsymbol{E}}: \epsilon^{e}
$$

which generalizes the isotropic definition $\tilde{\boldsymbol{\sigma}}=\boldsymbol{\sigma} /(1-D)$. In the further developments, initial elastic isotropy is assumed and one of the first things to do will be to define a fourth-order tensor $\underline{\boldsymbol{M}}$ in accordance with the use of a second-order damage variable (Equation (9)).

The classical damage models (see for instance section Unified framework for isotropic and anisotropic damage) lead to an effective Young's modulus in tension $\widetilde{E}_{\mathrm{t}}$ equal to the modulus in compression $\tilde{E}_{\mathrm{c}}$. This does not take into account the partial microdefects or micro-cracks closure effect which states that the damage measured in compression is smaller than the damage in tension (Figure 1), an effect simply represented by introducing a microdefects closure parameter $h_{\text {iso }}$ (isotropic damage case) or $h$ (anisotropic damage, see section Anisotropic damage with microdefects closure effect) in the coupling elasticity/damage in compression. If the effective Young's modulus in tension is still $E_{\mathrm{t}}=E(1-D)$, the effective Young's modulus in compression can be expressed as $E_{\mathrm{c}}=E\left(1-h_{\text {iso }} D\right)<E_{\mathrm{t}}$ where $0 \leq h_{\text {iso }}<1$. For metals, 
$h_{\text {iso }}$ remains close to 0.2 (Lemaitre, 1992) and will next be considered as a material parameter,

$$
h_{\text {iso }}=\frac{1-\tilde{E}_{\mathrm{t}} / E}{1-\tilde{E}_{\mathrm{c}} / E} \approx 0.2
$$

This phenomenon and its mechanical modeling are known as the unilateral conditions if the initial Young's modulus is fully recovered in compression (i.e., $\tilde{E}_{\mathrm{c}}=E$ or $h_{\text {iso }}=0$ ), as the quasi-unilateral (QU) conditions if the recovery is partial. Note that difficulties arise when $3 \mathrm{D}$ states of stresses are under consideration: what then is tension?; what then is compression?; how to ensure the continuity of the stress-strain law? Phenomenological answers will be given by using quadratic potentials built with positive and negative parts of tensors.

\section{UNIFIED FRAMEWORK FOR ISOTROPIC AND ANISOTROPIC DAMAGE}

Damage is represented by the second-order tensor $\boldsymbol{D}$. Isotropic damage is then the case $\boldsymbol{D}=\boldsymbol{D} \mathbf{1}$ with $\mathbf{1}$ being the second-order unit tensor.

\section{Gibbs Potential and the Effective Stress Concept}

Following Lemaitre et al. (2000), the Gibbs elastic potential $\rho \psi^{\star}$ (with $\rho$ as the density) is split in its deviatoric part affected by the tensorial damage variable $\boldsymbol{D}$ and its hydrostatic part affected by the scalar damage $\eta D_{H}=(\eta / 3)(\operatorname{tr} \mathbf{D})$

$$
\rho \psi^{\star}=\frac{1+v}{2 E} \operatorname{tr}\left((\mathbf{1}-\boldsymbol{D})^{-(1 / 2)} \boldsymbol{\sigma}^{D}(\mathbf{1}-\boldsymbol{D})^{-(1 / 2)} \boldsymbol{\sigma}^{D}\right)+\frac{3(1-2 \nu)}{2 E} \frac{\sigma_{H}^{2}}{1-\eta D_{H}},
$$

where $v$ is the initial Poisson's ratio and $\eta$ the necessary material parameter for the correct representation of experiments concerning the variations of the Poisson's ratio with damage. The parameter $\eta$ characterizes the sensitivity to hydrostatic stress and for metals it ranges between 2 and 3 . Note that to take a second-order tensor $\boldsymbol{A}$ at the power $-1 / 2$ consists (i) of making $\boldsymbol{A}$ diagonal through the change of base matrix $\boldsymbol{P}, \boldsymbol{A}_{\mathrm{diag}}=\boldsymbol{P}^{-1} \boldsymbol{A P}$, (ii) of taking the power $-1 / 2$ of the diagonal terms defining $\boldsymbol{A}_{\text {diag }}^{-1 / 2}$, (iii) of turning back the tensor in its initial base $\boldsymbol{A}^{-1 / 2}=\boldsymbol{P} \boldsymbol{A}_{\mathrm{diag}}^{-1 / 2} \boldsymbol{P}^{-1}$. Note that the same change of base procedure will be used to define the 
absolute value of a second-order tensor introduced (see section Anisotropic Damage).

In Equation (4) $\sigma^{D}$ denotes the stress deviator and $\sigma_{H}$ the hydrostatic stress,

$$
\boldsymbol{\sigma}^{D}=\boldsymbol{\sigma}-\sigma_{H} \mathbf{1}, \quad \sigma_{H}=\frac{1}{3} \operatorname{tr} \boldsymbol{\sigma}
$$

and for further derivations, the tensor

$$
\boldsymbol{H}=(\mathbf{1}-\boldsymbol{D})^{-(1 / 2)}
$$

is defined as the effective damage tensor.

The law of elasticity derives from Gibbs potential,

$$
\boldsymbol{\epsilon}^{e}=\rho \frac{\partial \psi^{\star}}{\partial \boldsymbol{\sigma}}=\frac{1+v}{E} \tilde{\boldsymbol{\sigma}}-\frac{v}{E} t r \tilde{\boldsymbol{\sigma}} \mathbf{1}
$$

It naturally introduces a symmetric effective stress tensor which does not depend upon the elasticity parameters and which will be used for the coupling with plasticity,

$$
\tilde{\boldsymbol{\sigma}}=\left(\boldsymbol{H} \boldsymbol{\sigma}^{D} \boldsymbol{H}\right)^{D}+\frac{\sigma_{H}}{1-\eta D_{H}} \mathbf{1}
$$

and as in the isotropic case the components of the damage tensor may be obtained from the elasticity changes.

Equation (8) defines the fourth order tensor $\underline{M}$ according to the representation of the damage through the second order tensor $\boldsymbol{D}$,

$$
\underline{\boldsymbol{M}}=\boldsymbol{H} \underline{\otimes} \boldsymbol{H}-\frac{1}{3}\left[\boldsymbol{H}^{2} \otimes \mathbf{1}+\mathbf{1} \otimes \boldsymbol{H}^{2}\right]+\frac{1}{9}\left(t r \boldsymbol{H}^{2}\right) \mathbf{1} \otimes \mathbf{1}+\frac{1}{3\left(1-\eta D_{H}\right)} \mathbf{1} \otimes \mathbf{1}
$$

where $\otimes$ is the tensorial product $(\boldsymbol{A} \otimes \boldsymbol{B})_{i j k l}=A_{i j} B_{k l}$ and where the special tensorial product $\boldsymbol{H} \underline{\otimes} \boldsymbol{H}$ applied on symmetric second order tensor $\boldsymbol{H}$ stands for $(\boldsymbol{H} \underline{\otimes} \boldsymbol{H})_{i j k l}=H_{i k} \bar{H}_{j l}$.

The relationship between stress and effective stress tensors can be inverted in:

$$
\boldsymbol{\sigma}=\underline{\boldsymbol{M}}^{-1}: \tilde{\boldsymbol{\sigma}}=\boldsymbol{H}^{-1} \tilde{\boldsymbol{\sigma}} \boldsymbol{H}^{-1}-\frac{(\mathbf{1}-\boldsymbol{D}): \tilde{\boldsymbol{\sigma}}}{3\left(1-D_{H}\right)}(\mathbf{1}-\boldsymbol{D})+\left(1-\eta D_{H}\right) \tilde{\sigma}_{H} \mathbf{1}
$$


with $\boldsymbol{H}^{-1}=(\mathbf{1}-\boldsymbol{D})^{1 / 2}$ and

$$
\underline{\boldsymbol{M}}^{-1}=\boldsymbol{H}^{-1} \underline{\otimes}^{-1}-\frac{(\mathbf{1}-\boldsymbol{D}) \otimes(\mathbf{1}-\boldsymbol{D})}{3\left(1-D_{H}\right)}+\frac{1}{3}\left(1-\eta D_{H}\right) \mathbf{1} \otimes \mathbf{1}
$$

\section{Evolution Laws}

The evolution laws concern the dissipative mechanisms such as plasticity and damage. Refer to Lemaitre and Chaboche's work (1985) for the corresponding thermodynamics framework (see also Lemaitre and Demorat, 2005).

ISOTROPIC DAMAGE

Lemaitre's damage evolution law of damage governed by plasticity (through $p$ ) and enhanced by the strain energy (through the strain energy release rate density $\mathrm{Y}$ ),

$$
\dot{D}=\left(\frac{Y}{S}\right)^{s} \dot{p} \quad Y=\frac{1}{2} \underline{E}: \epsilon^{e}: \epsilon^{e}=\frac{\tilde{\sigma}_{\mathrm{eq}}^{2} R_{v}}{2 E}
$$

makes it possible to model damage growth for ductile failure, fatigue, creep or creep-fatigue (Sermage et al., 2000; Desmorat, 2000a,b; Lemaitre and Demorat, 2005). Two damage parameters $S, s$ are introduced and $p=\int \sqrt{(2 / 3) \dot{\boldsymbol{\epsilon}}^{p}: \dot{\boldsymbol{\epsilon}}^{p}} \mathrm{~d} t$ is the accumulated plastic strain. The stress triaxiality effect is taken into account through the triaxiality function $R_{v}$,

$$
R_{v}=\frac{2}{3}(1+v)+3(1-2 v)\left(\frac{\sigma_{H}}{\sigma_{\mathrm{eq}}}\right)^{2}
$$

\section{ANISOTROPIC DAMAGE}

The damage evolution law (Lemaitre et al., 2000; Lemaitre and Demorat, 2001),

$$
\dot{\boldsymbol{D}}=\left(\frac{\bar{Y}}{S}\right)^{s}\left|\dot{\boldsymbol{\epsilon}}^{p}\right|, \quad \text { or } \quad \dot{D}_{i j}=\left(\frac{\bar{Y}}{S}\right)^{s}\left|\dot{\boldsymbol{\epsilon}}^{p}\right|_{i j}
$$

generalizes the isotropic law (12) to anisotropic damage induced by plasticity. The principal directions of the damage rate coincide with those of the plastic strain rate. The notation $|\cdot|$ applied to a tensor means the absolute value of 
the principal values, i.e. (i) make the tensor diagonal, (ii) take the absolute value of its diagonal terms, and (iii) turn it back in its initial base. Examples for tension and shear are given next. The dependency on the strain energy is preserved by defining $\bar{Y}$ as the effective strain energy $\int \tilde{\sigma}_{i j} d \epsilon_{i j}^{e}$,

$$
\bar{Y}=\frac{1}{2} \underline{E}: \epsilon^{e}: \epsilon^{e}=\frac{1}{2} \tilde{\sigma}_{i j} \epsilon_{i j}^{e}=\frac{\tilde{\sigma}_{\mathrm{eq}}^{2} \tilde{R}_{v}}{2 E}
$$

This introduces the effective triaxiality function:

$$
\tilde{R}_{v}=\frac{2}{3}(1+v)+3(1-2 v)\left(\frac{\tilde{\sigma}_{H}}{\tilde{\sigma}_{\mathrm{eq}}}\right)^{2}
$$

In uniaxial tension, one has:

$$
\dot{\boldsymbol{\epsilon}}^{p}=\left[\begin{array}{ccc}
\dot{\epsilon}_{p} & 0 & 0 \\
0 & -\frac{1}{2} \dot{\epsilon}_{p} & 0 \\
0 & 0 & -\frac{1}{2} \dot{\epsilon}_{p}
\end{array}\right]
$$

Then,

$$
\dot{\boldsymbol{D}}=\left(\frac{\bar{Y}}{S}\right)^{s}\left|\dot{\epsilon}_{p}\right|\left[\begin{array}{ccc}
1 & 0 & 0 \\
0 & \frac{1}{2} & 0 \\
0 & 0 & \frac{1}{2}
\end{array}\right]
$$

which ends up with the feature $D_{1}=2 D_{2}$ of damage twice as large in the tension direction than in the transverse directions as observed experimentally for metals (Lemaitre et al., 2000).

In shear:

$$
\dot{\boldsymbol{\epsilon}}^{p}=\left[\begin{array}{ccc}
0 & \dot{\epsilon}_{12}^{p} & 0 \\
\dot{\epsilon}_{12}^{p} & 0 & 0 \\
0 & 0 & 0
\end{array}\right]
$$

Make $\dot{\epsilon}^{p}$ diagonal

$$
P^{-1} \dot{\boldsymbol{\epsilon}}^{p} P=\left[\begin{array}{ccc}
\dot{\epsilon}_{12}^{p} & 0 & 0 \\
0 & -\dot{\epsilon}_{12}^{p} & 0 \\
0 & 0 & 0
\end{array}\right]
$$


Take the absolute value

$$
\left|P^{-1} \dot{\boldsymbol{\epsilon}}^{p} P\right|=\left[\begin{array}{ccc}
\left|\dot{\epsilon}_{12}^{p}\right| & 0 & 0 \\
0 & \left|\dot{\epsilon}_{12}^{p}\right| & 0 \\
0 & 0 & 0
\end{array}\right]
$$

Then,

$$
\dot{\boldsymbol{D}}=\left[\begin{array}{ccc}
\dot{D} & 0 & 0 \\
0 & \dot{D} & 0 \\
0 & 0 & 0
\end{array}\right], \quad \dot{D}=\left(\frac{\bar{Y}}{S}\right)^{s}\left|\dot{\epsilon}_{12}^{p}\right|
$$

which corresponds to isotropic damage in the shear plane.

\section{DAMAGE THRESHOLD}

Furthermore, the damage exists only above a threshold $p_{D}$ written in terms of accumulated plastic strain ( $p_{D}$ is then a material parameter) or in terms of stored energy density ( $p_{D}$ is then loading dependent, Lemaitre and Demorat, 2005),

$$
\dot{\boldsymbol{D}}=0 \quad \text { if } \quad p \leq p_{D}
$$

\section{MESOCRACK INITIATION}

A mesocrack initiates when the damage reaches a critical value $D_{\mathrm{c}}$ which is also a material parameter ( $D_{\mathrm{c}}$ ranges between 0.2 and 0.5 for metals). This value corresponds to the instability under loading in the plane (of normal $\mathbf{n}$ ) in which the density of microcracks is maximum. This density is defined by the norm of the damage vector $D_{i j} n_{j}$ or by the larger principal value of the damage. Then,

$$
\sup _{I} D_{I}=D_{\mathrm{c}} \rightarrow \text { mesocrack initiation }
$$

\section{FULL COUPLING WITH PLASTICITY}

For metals, damage is governed by plasticity and the effective stress concept is used for the coupling plasticity/damage, i.e. the stress $\sigma$ is replaced by the effective stress $\tilde{\boldsymbol{\sigma}}$ in von Mises yield criterion,

$$
f=\tilde{\sigma}_{\mathrm{eq}}-R-\sigma_{\mathrm{y}}, \quad \tilde{\sigma}_{\mathrm{eq}}=\sqrt{\frac{3}{2} \tilde{\boldsymbol{\sigma}}^{D}: \tilde{\boldsymbol{\sigma}}^{D}}
$$


where $f$ is the yield function and $\sigma_{\mathrm{y}}$ the yield stress: $f<0$ implies elasticity and plasticity coupled with damage occurs for $f=0, \dot{f}=0$. Isotropic hardening $(R)$ is assumed.

The law of plasticity coupled with damage derives from the potential $f$ through the normality rule,

$$
\begin{gathered}
\dot{\boldsymbol{\epsilon}}^{p}=\dot{\lambda} \frac{\partial f}{\partial \boldsymbol{\sigma}}=\dot{\lambda} \frac{3}{2} \frac{\left(\boldsymbol{H} \tilde{\boldsymbol{\sigma}}^{D} \boldsymbol{H}\right)^{D}}{\tilde{\sigma}_{\mathrm{eq}}} \\
\dot{r}=-\dot{\lambda} \frac{\partial f}{\partial R}=\dot{\lambda}=\dot{p} \frac{\tilde{\sigma}_{\mathrm{eq}}}{\left(\boldsymbol{H} \tilde{\boldsymbol{\sigma}}^{D} \boldsymbol{H}\right)_{\mathrm{eq}}}
\end{gathered}
$$

with $\dot{\lambda}$ the plastic multiplier determined by the consistency condition $f=0$, $\dot{f}=0$. Equation (27) defines the internal variable $r$ associated with $R$ as $\int \dot{\mathrm{r}} \mathrm{\textrm {d }} t=\int \dot{\mathrm{\lambda}} \mathrm{d} t$ so that the isotropic hardening law is the function $R=R(r)$. Note that as long as there is no damage the equality $r=p$ stands and the classical writing $R=R(p)$ is recovered.

\section{Isotropic Model as a Limiting Case of the Anisotropic Damage Model}

To consider damage evolution law

$$
\dot{\boldsymbol{D}}=\left(\frac{\bar{Y}}{S}\right)^{s} \dot{p} \mathbf{1}=\dot{D} \mathbf{1}
$$

instead of the anisotropic law (14) allows recovery of classical elastoplasticity coupled with isotropic damage as Equation (6) simplifies in

$$
\boldsymbol{H}=\frac{1}{\sqrt{1-D}} \mathbf{1}
$$

With $\eta=1$, Equation (8) reduces to the classical definition of the effective stress:

$$
\tilde{\boldsymbol{\sigma}}=\frac{\sigma}{1-D}
$$

and Equations (26) and (27) recover the isotropic case (Lemaitre and Chaboche, 1985),

$$
\dot{\boldsymbol{\epsilon}}^{p}=\frac{\dot{\lambda}}{1-D} \frac{3}{2} \frac{\sigma^{D}}{\tilde{\sigma}_{\mathrm{eq}}}, \quad \dot{r}=\dot{\lambda}=(1-D) \dot{p}
$$




\section{MODELING MICRODEFECTS' CLOSURE EFFECT}

The state variable $D$ or $\boldsymbol{D}$ represent the state of microdefects (microcracks or microvoids) present at a scale smaller than the representative volume element scale of continuum mechanics. As long as such microcracks do not propagate (elastic regime corresponding to the state laws), the state of damage remains constant and does not depend then on the loading, its kind and sign. This means that no other thermodynamics damage variable is needed to model the (quasi) unilateral conditions. The models exposed and proposed thereafter fulfill this last point.

The continuity of the stress-strain law $\sigma_{i j}\left(\epsilon_{i j}^{e}\right)$ and of the law $Y(D)$ will naturally be obtained by considering a potential which can be continuously differentiated (Ladevèze, 1983).

\section{Isotropic Damage with Microdefects' Closure Effect}

The isotropic case has been solved by Ladevèze and Lemaitrè, 1984 (see also Lemaitre, 1992), who consider the potential

$$
\rho \psi_{e}^{\star}=\frac{1+v}{2 E}\left[\frac{\langle\boldsymbol{\sigma}\rangle_{+}:\langle\boldsymbol{\sigma}\rangle_{+}}{1-D}+\frac{\langle\boldsymbol{\sigma}\rangle_{-}:\langle\boldsymbol{\sigma}\rangle_{-}}{1-h_{\mathrm{iso}} D}\right]-\frac{v}{2 E}\left[\frac{\langle\operatorname{tr} \boldsymbol{\sigma}\rangle_{+}^{2}}{1-D}+\frac{\langle\operatorname{tr} \boldsymbol{\sigma}\rangle_{-}^{2}}{1-h_{\mathrm{iso}} D}\right]
$$

which can be continuously differentiated and where $\langle.\rangle_{+}$(respectively $\langle.\rangle_{-}$) denotes the positive part (respectively negative part) in terms of principal components for a tensor. No new damage variable is considered and a microdefects closure parameter $h_{\text {iso }}$, material dependent, is introduced $\left(0 \leq h_{\text {iso }} \leq 1\right)$.

\section{ELASTICITY COUPLED WITH DAMAGE}

The elasticity law is then

$$
\boldsymbol{\epsilon}^{e}=\rho \frac{\partial \psi_{e}^{\star}}{\partial \boldsymbol{\sigma}}=\frac{1+v}{2 E}\left[\frac{\langle\boldsymbol{\sigma}\rangle_{+}}{1-D}+\frac{\langle\boldsymbol{\sigma}\rangle_{-}}{1-h_{\mathrm{iso}} D}\right]-\frac{v}{E}\left[\frac{\langle\operatorname{tr} \boldsymbol{\sigma}\rangle_{+}}{1-D}+\frac{\langle\operatorname{tr} \boldsymbol{\sigma}\rangle_{-}}{1-h_{\text {iso }} D}\right] \mathbf{1}
$$

It defines an effective stress (Lemaitre 1992):

$$
\tilde{\boldsymbol{\sigma}}=\frac{\langle\boldsymbol{\sigma}\rangle_{+}}{1-D}+\frac{\langle\boldsymbol{\sigma}\rangle_{-}}{1-h_{\mathrm{iso}} D}+\frac{v}{1-2 v}\left(\frac{\mathbf{1}:\langle\boldsymbol{\sigma}\rangle_{+}-\langle\operatorname{tr} \boldsymbol{\sigma}\rangle_{+}}{1-D}+\frac{1:\langle\boldsymbol{\sigma}\rangle_{-}-\langle\operatorname{tr} \boldsymbol{\sigma}\rangle_{-}}{1-h_{\mathrm{iso}} D}\right) \mathbf{1}
$$


The strain energy release rate density is the variable associated with $D$,

$$
\begin{aligned}
Y=\rho \frac{\partial \psi_{e}^{\star}}{\partial Y}= & \frac{1+v}{2 E}\left[\frac{\langle\boldsymbol{\sigma}\rangle_{+}:\langle\boldsymbol{\sigma}\rangle_{+}}{(1-D)^{2}}+h_{\text {iso }} \frac{\langle\boldsymbol{\sigma}\rangle_{-}:\langle\boldsymbol{\sigma}\rangle_{-}}{\left(1-h_{\text {iso }} D\right)^{2}}\right] \\
& -\frac{v}{2 E}\left[\frac{\langle t r \boldsymbol{\sigma}\rangle_{+}^{2}}{(1-D)^{2}}+h_{\text {iso }} \frac{\langle t r \boldsymbol{\sigma}\rangle_{-}^{2}}{\left(1-h_{\text {iso }} D\right)^{2}}\right]
\end{aligned}
$$

The formulation extends the concept of tension and compression to $3 \mathrm{D}$ loading by use of the positive and negative parts of the stress tensor.

- For the monotonic tensile case ( $\sigma>0$ ), Equations (33) and (35) read:

$$
\epsilon_{e}=\frac{\sigma}{E(1-D)} \quad Y=\frac{\sigma^{2}}{2 E(1-D)^{2}}
$$

which gives $\tilde{E}_{t}=E(1-D)$.

- For the monotonic compression case $(\sigma<0)$,

$$
\epsilon_{e}=\frac{\sigma}{E\left(1-h_{\text {iso }} D\right)} \quad Y=h_{\text {iso }} \frac{\sigma^{2}}{2 E\left(1-h_{\text {iso }} D\right)^{2}}
$$

gives $\tilde{E}_{c}=E\left(1-h_{\text {iso }} D\right)$.

Then Equation (3) is recovered and gives $h_{\text {iso }}=0.2$ as standard value for the material parameter $h_{\text {iso }}$.

\section{COUPLING WITH PLASTICITY}

The effective stress concept, judicious to write the coupling with plasticity without unilateral conditions, cannot be used coupled with the microdefects closure effect (Liénard, 1989): the yield surface $f=\left(\tilde{\boldsymbol{\sigma}}^{D}-\boldsymbol{X}^{D}\right)_{\mathrm{eq}}-R-\sigma_{\mathrm{y}}$ with both isotropic and kinematic hardening $R$ and $\boldsymbol{X}$ and with $\tilde{\boldsymbol{\sigma}}$ given by Equation (34) is nonconvex with angular points (points A and B of Figure 1). Here $\boldsymbol{X}$ denotes the kinematic hardening or back stress. Such a yield surface cannot be continuously differentiated because of the terms $\boldsymbol{X}:\langle\boldsymbol{\sigma}\rangle_{+}, \boldsymbol{X}:\langle\boldsymbol{\sigma}\rangle_{-}, \operatorname{tr}\langle\boldsymbol{\sigma}\rangle_{+}, \operatorname{tr}\langle\boldsymbol{\sigma}\rangle_{-}$, within the developed expression of $(\tilde{\boldsymbol{\sigma}}-\boldsymbol{X})_{\mathrm{eq}}$.

To get a convex yield criterion one proposes the following expression for $f$,

$$
f=\sqrt{\frac{3}{2} \operatorname{tr}\left[\left\langle\tilde{\boldsymbol{\sigma}}^{D+}-\boldsymbol{X}\right\rangle_{+}^{2}+\left\langle\tilde{\boldsymbol{\sigma}}^{D-}-\boldsymbol{X}\right\rangle_{-}^{2}\right]}+\alpha\left(1-h_{\mathrm{iso}}\right) D \operatorname{tr} \boldsymbol{\sigma}-R-\sigma_{\mathrm{y}}
$$

where

$$
\tilde{\boldsymbol{\sigma}}^{D+}=\frac{\boldsymbol{\sigma}^{D}}{1-D}, \quad \tilde{\boldsymbol{\sigma}}^{D-}=\frac{\boldsymbol{\sigma}^{D}}{1-h_{\mathrm{iso}} D}
$$




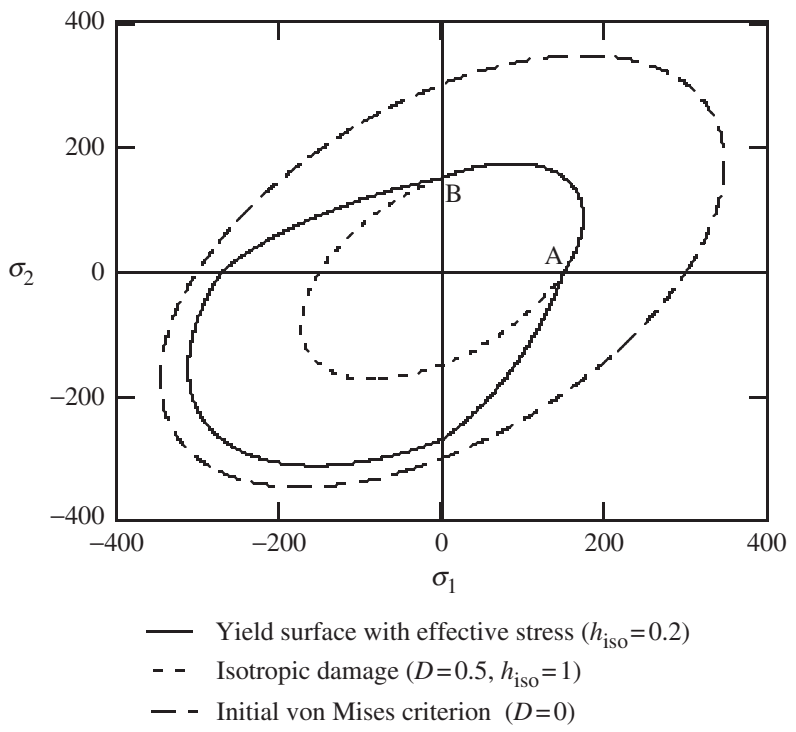

Figure 1. Non convex yield criterion $\left(\sigma_{y}+R=300 \mathrm{MPa}, h_{\text {iso }}=0.2, X=0\right)$.

The criterion (38) has a Drucker-Prager term when the unilateral condition is activated $\left(h_{\text {iso }} \neq 0\right)$ and when the material is damaged. When $D=0$, von Mises yield criterion is recovered. $\alpha$ is a material parameter but note that to set up $\alpha=1-h_{\text {iso }}$ leads to a yield surface very close (but convex) to the yield surface obtained by use of the effective stress (34) (Figure 2).

Both the differentiability and the convexity of the proposed yield surface are obtained by the consideration of quadratic terms $\operatorname{tr}\left[\left\langle\tilde{\boldsymbol{\sigma}}^{D+}-\boldsymbol{X}\right\rangle_{+ \text {or }-}^{2}\right]$ within $f$ (Ladevèze, 1983; Desmorat, 2002). But only a complete experimental campaign will show the relevance of the proposed criterion and of the necessity to take into account or not the microdefects' closure effects within the yield function.

\section{DAMAGE EVOLUTION}

Still consider Lemaitre's damage evolution law $\dot{D}=(Y / S)^{s} \dot{p}$, but the strain energy release rate $Y$ now depends on the parameter $h_{\text {iso }}$ (Equation (35)). For uniaxial loading, the damage law reads

$$
\begin{aligned}
& \dot{D}=\left(\frac{\sigma^{2}}{2 E(1-D)^{2}}\right)^{s} \dot{p} \quad \text { in tension } \\
& \dot{D}=\left(\frac{h_{\text {iso }} \sigma^{2}}{2 E\left(1-h_{\text {iso }} D\right)^{2}}\right)^{s} \dot{p} \text { in compression }
\end{aligned}
$$




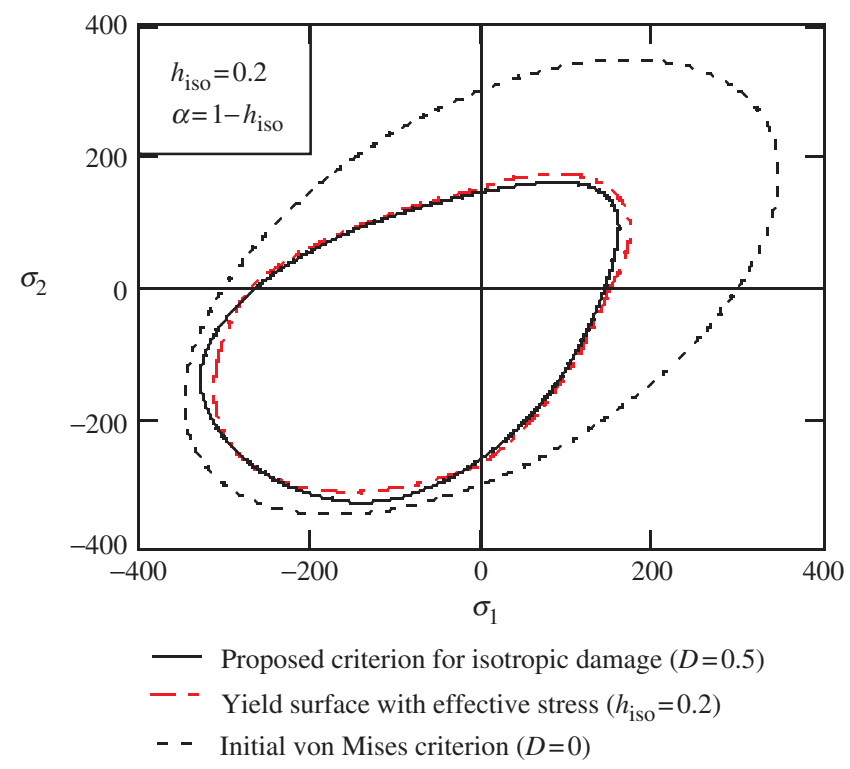

Figure 2. Convex yield criterion with isotropic damage $\left(\sigma_{y}+R=300 \mathrm{MPa}, h_{\text {iso }}=0.2, X=0\right.$, $D=0.5)$.

and takes into account an evolution of the damage slower in compression than in tension as for the same stress (in absolute value):

$$
\dot{D}_{\text {compression }} \approx h_{\text {iso }}^{s} \dot{D}_{\text {tension }}
$$

\section{Anisotropic Damage with Microdefects Closure Effect}

In order to model the unilateral (or QU) conditions, Chaboche (1993) and Halm and Dragon (1998) introduce from homogenization considerations an additional fourth-order damage tensor $\underline{\hat{D}}$ in the thermodynamics potential, a tensor which is not an extra variable but which is built from the principal directions $\vec{v}^{I}$ and damages $D_{I}$ of the second-order damage tensor $\boldsymbol{D}$,

$$
\underline{\hat{\boldsymbol{D}}}=\sum_{I=1}^{6} D_{I} \mathcal{H}\left(-\vec{v}^{I} \cdot \boldsymbol{\epsilon} \cdot \vec{v}^{I}\right) \vec{v}^{I} \otimes \vec{v}^{I} \otimes \vec{v}^{I} \otimes \vec{v}^{I}
$$

where $\mathcal{H}$ is the Heaviside function. The tensor $\underline{\hat{\boldsymbol{D}}}$ is loading dependent due to the term $\mathcal{H}\left(-\vec{v}_{I} \cdot \epsilon \cdot \vec{v}_{I}\right)$. A strain formulation is used and $\underline{\hat{D}}$ is introduced 
as $\boldsymbol{\epsilon}: \underline{\hat{D}}: \boldsymbol{\epsilon}$ within Helmholtz free energy. The derivative $\partial(\boldsymbol{\epsilon}: \underline{\hat{D}}: \boldsymbol{\epsilon}) / \partial \boldsymbol{\epsilon}$ is continuous and leads to a tensorial continuous stress-strain law. The formulation automatically defines what is tension and what is compression for any 3D state of stress. The drawback is that the calculation of the tensorial strain energy release rate is not possible as the term $\partial \underline{\hat{\boldsymbol{D}}} / \partial \boldsymbol{D}$ (including $\partial \vec{v}_{I} / \partial \boldsymbol{D}$ ) cannot be calculated for arbitrary and possibly rotating damage principal directions. In other terms the expression $\boldsymbol{\epsilon}: \underline{\hat{\boldsymbol{D}}}: \boldsymbol{\epsilon}$ cannot be continuously differentiated.

A more general approach but farther from the microscopic observations of the damage mechanisms is developed by Ladevèze $(1983,1995)$, approach mostly applied to composite materials. Four damage variables $\boldsymbol{H}_{1}, \boldsymbol{H}_{2}$ (tensorial) and $d_{1}, d_{2}$ (scalar) are introduced. The general form of Gibbs potential is then written as

$$
\rho \psi^{\star}=a_{1} \operatorname{tr}\left[\boldsymbol{H}_{1} \boldsymbol{\sigma}_{+} \boldsymbol{H}_{1} \boldsymbol{\sigma}_{+}+\boldsymbol{H}_{2} \boldsymbol{\sigma}_{-} \boldsymbol{H}_{2} \boldsymbol{\sigma}_{-}\right]+a_{2}\left[\frac{\langle t r \boldsymbol{\sigma}\rangle_{+}^{2}}{1-d_{1}}+\frac{\langle t r \boldsymbol{\sigma}\rangle_{-}^{2}}{1-d_{2}}\right]
$$

A 'special' positive part $\boldsymbol{\sigma}_{+}$of $\boldsymbol{\sigma}$ (respectively negative part $\boldsymbol{\sigma}_{-}$) is built with the positive (respectively negative) eigenvalues and with the corresponding eigenvectors of the matrix $\left(\boldsymbol{H}_{1} \boldsymbol{\sigma}\right)$ (respectively $\left(\boldsymbol{H}_{2} \boldsymbol{\sigma}\right)$ ) in order to make $\rho \psi^{\star}$ able to be continuously differentiated and to have:

$$
\mathrm{d} \operatorname{tr}\left[\boldsymbol{H}_{1} \boldsymbol{\sigma}_{+} \boldsymbol{H}_{1} \boldsymbol{\sigma}_{+}\right]=2 \operatorname{tr}\left[\boldsymbol{H}_{1} \boldsymbol{\sigma}_{+} \boldsymbol{H}_{1} \mathrm{~d} \boldsymbol{\sigma}\right]+2 \operatorname{tr}\left[\boldsymbol{\sigma}_{+} \boldsymbol{H}_{1} \boldsymbol{\sigma}_{+} \mathrm{d} \boldsymbol{H}_{1}\right]
$$

The expression for $\boldsymbol{\sigma}_{+}$and $\boldsymbol{\sigma}_{-}$are not given here as they are detailed in the next section for tensor $\boldsymbol{\sigma}^{D}$.

The thermodynamics variables associated with $\boldsymbol{H}_{1}, \boldsymbol{H}_{2}, d_{1}, d_{2}$ can then be calculated,

$$
\begin{aligned}
& Z_{1}=\rho \frac{\partial \psi^{\star}}{\partial \boldsymbol{H}_{1}}=2 a_{1} \boldsymbol{\sigma}_{+} \boldsymbol{H}_{1} \boldsymbol{\sigma}_{+}, \quad Z_{2}=\rho \frac{\partial \psi^{\star}}{\partial \boldsymbol{H}_{2}}=2 a_{1} \boldsymbol{\sigma}_{-} \boldsymbol{H}_{2} \boldsymbol{\sigma}_{-} \\
& Y_{1}=\rho \frac{\partial \psi^{\star}}{\partial d_{1}}=a_{2} \frac{\langle t r \boldsymbol{\sigma}\rangle_{+}^{2}}{\left(1-d_{1}\right)^{2}}, \quad Y_{2}=\rho \frac{\partial \psi^{\star}}{\partial d_{2}}=a_{2} \frac{\langle t r \boldsymbol{\sigma}\rangle_{-}^{2}}{\left(1-d_{2}\right)^{2}}
\end{aligned}
$$

as well as the dissipation due to damage:

$$
\mathcal{D}=\operatorname{tr}\left(\boldsymbol{Z}_{1} \dot{\boldsymbol{H}}_{1}\right)+\operatorname{tr}\left(\boldsymbol{Z}_{2} \dot{\boldsymbol{H}}_{2}\right)+Y_{1} \dot{d}_{1}+Y_{2} \dot{d}_{2}
$$

\section{ELASTICITY COUPLED WITH ANISOTROPIC DAMAGE}

Let us apply Ladevèze's framework to build a Gibbs potential for anisotropic damage which models the QU conditions. 
In a consistent manner with the initial anisotropic damage model, it is written as:

$$
\begin{aligned}
\rho \psi^{\star}= & \frac{1+v}{2 E} \operatorname{tr}\left(\boldsymbol{H}^{p} \sigma_{+}^{D} \boldsymbol{H}^{p} \sigma_{+}^{D}+\boldsymbol{H}^{n} \sigma_{-}^{D} \boldsymbol{H}^{n} \sigma_{-}^{D}\right) \\
& +\frac{3(1-2 v)}{2 E}\left(\frac{\left\langle\sigma_{H}\right\rangle_{+}^{2}}{1-\eta D_{H}}+\frac{\left\langle\sigma_{H}\right\rangle_{-}^{2}}{1-h \eta D_{H}}\right)
\end{aligned}
$$

A crack closure parameter $h$ is introduced as for isotropy and one defines here:

$$
\boldsymbol{H}^{p}=(\mathbf{1}-\boldsymbol{D})^{-1 / 2}, \quad \boldsymbol{H}^{n}=(\mathbf{1}-h \boldsymbol{D})^{-1 / 2}
$$

A single damage variable is used but which acts fully through $\boldsymbol{D}$ for 'tension' and partially through $h \boldsymbol{D}$ in 'compression'. In order to keep the differentiability of Gibbs energy, the positive (respectively negative) parts are taken with respect to the deviatoric stress tensor; $\boldsymbol{\sigma}_{+}^{D}$ is built with the eigenvalues $\lambda^{I}$ and the corresponding eigenvectors $\boldsymbol{T}^{I}$ of $\left(\boldsymbol{H}^{p} \boldsymbol{\sigma}^{D}\right)$ in such a way that

$$
\mathrm{d}\left[\frac{1}{2} \operatorname{tr}\left(\boldsymbol{H}^{p} \boldsymbol{\sigma}_{+}^{D} \boldsymbol{H}^{p} \boldsymbol{\sigma}_{+}^{D}\right)\right]=\operatorname{tr}\left(\boldsymbol{H}^{p} \boldsymbol{\sigma}_{+}^{D} \boldsymbol{H}^{p} \mathrm{~d} \boldsymbol{\sigma}^{D}\right)+\operatorname{tr}\left(\boldsymbol{\sigma}_{+}^{D} \boldsymbol{H}^{p} \boldsymbol{\sigma}_{+}^{D} \mathrm{~d} \boldsymbol{H}^{p}\right)
$$

$T^{I}$ and $\lambda^{I}$ are given by the eigenvalue problem

$$
\boldsymbol{\sigma}^{D} \boldsymbol{T}^{I}=\lambda^{I}\left(\boldsymbol{H}^{p}\right)^{-1} \boldsymbol{T}^{I} \text { (without summation) }
$$

in which the normalization $\boldsymbol{T}^{I T}\left(\boldsymbol{H}^{p}\right)^{-1} \boldsymbol{T}^{J}=\delta_{I J}$ is made $\left((.)^{T}\right.$ denotes the transpose). The eigenvalues $\lambda^{I}$ are real because $\boldsymbol{\sigma}^{D}$ is symmetric and $\left(\boldsymbol{H}^{p}\right)^{-1}$ is positive defined and symmetric. Then the deviatoric stress tensor may be expressed as

$$
\boldsymbol{\sigma}^{D}=\sum_{I=1}^{3}\left[\left(\boldsymbol{H}^{p}\right)^{-1} \boldsymbol{T}^{I}\right]\left[\left(\boldsymbol{H}^{p}\right)^{-1} \boldsymbol{T}^{I}\right]^{T} \lambda^{I}
$$

and the positive part $\boldsymbol{\sigma}_{+}^{D}$ is, therefore, defined as

$$
\boldsymbol{\sigma}_{+}^{D}=\sum_{i=1}^{3}\left[\left(\boldsymbol{H}^{p}\right)^{-1} T_{i}\right]\left[\left(\boldsymbol{H}^{p}\right)^{-1} T_{i}\right]^{T}\left\langle\lambda_{i}\right\rangle_{+}
$$

The same work is made for the negative part $\boldsymbol{\sigma}_{-}^{D}$ (with $\boldsymbol{H}^{n}$ ). 
The elasticity law derives from Gibbs potential:

$$
\begin{aligned}
\boldsymbol{\epsilon}^{e}= & \rho \frac{\partial \psi^{\star}}{\partial \boldsymbol{\sigma}} \\
= & \frac{1+v}{E}\left[\left(\boldsymbol{H}^{p} \boldsymbol{\sigma}_{+}^{D} \boldsymbol{H}^{p}\right)^{D}+\left(\boldsymbol{H}^{n} \boldsymbol{\sigma}_{-}^{D} \boldsymbol{H}^{m}\right)^{D}\right] \\
& +\frac{1-2 v}{E}\left[\frac{\left\langle\sigma_{H}\right\rangle_{+}}{1-\eta D_{H}}+\frac{\left\langle\sigma_{H}\right\rangle_{-}}{1-h \eta D_{H}}\right] \mathbf{1} \\
= & \frac{1+v}{E} \tilde{\boldsymbol{\sigma}}-\frac{v}{E} t r \tilde{\boldsymbol{\sigma}} \mathbf{1}
\end{aligned}
$$

It is continuous and it defines the effective stress as:

$$
\begin{aligned}
\tilde{\boldsymbol{\sigma}}= & \left(\boldsymbol{H}^{p} \boldsymbol{\sigma}_{+}^{D} \boldsymbol{H}^{p}\right)^{D}+\left(\boldsymbol{H}^{n} \boldsymbol{\sigma}_{-}^{D} \boldsymbol{H}^{n}\right)^{D} \\
& +\left[\frac{\left\langle\sigma_{H}\right\rangle_{+}}{1-\eta D_{H}}-\frac{\left\langle-\sigma_{H}\right\rangle_{-}}{1-h \eta D_{H}}\right] \mathbf{1}
\end{aligned}
$$

which takes into account the QU effect.

In order to keep the differentiability of the thermodynamics potential the positive (negative) parts are now taken with respect to the deviatoric stress tensor. This means that the parameter $h$ affects both tension and compression (in any uniaxial tensile case $\sigma^{D}$ has positive and negative components); therefore the model is quite complex even for the suppositively simple tensile test. Furthermore, the value of $h$ depends on the model itself: for a given material, $h_{\text {iso }}$ for the isotropic damage model is different from $h$ identified for the anisotropic damage model. The good thing is that the common value for metals with $h_{\text {iso }}=0.2$ in the isotropic case corresponds to a partial stiffness recovery gained with $h=0$ in the anisotropic case. This is due to the fact that for the anisotropic model the positive parts are taken on the deviatoric stresses instead of the stresses: in compression, the stress deviator still has positive terms on which the damage $\boldsymbol{D}$ fully acts even if $h=0$.

\section{COUPLING WITH PLASTICITY}

In order to define convex yield criterion, let us take as yield function:

$$
\begin{aligned}
f= & \sqrt{\frac{3}{2} \operatorname{tr}\left[\left\langle\tilde{\boldsymbol{\sigma}}^{D+}-\boldsymbol{X}^{D}\right\rangle_{+}^{2}+\left\langle\tilde{\boldsymbol{\sigma}}^{D-}-\boldsymbol{X}^{D}\right\rangle_{-}^{2}\right]} \\
& +\alpha(1-h) d_{H} \operatorname{tr} \boldsymbol{\sigma}-R-\sigma_{\mathrm{y}}
\end{aligned}
$$


where

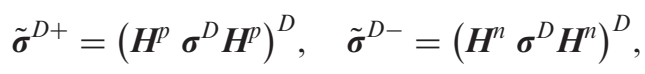

and where $\alpha$ is a material parameter. The yield surface $f=0$ is chosen to coincide with the previous surface for isotropic damage, i.e. when $\boldsymbol{D}=D \mathbf{1}$ and $h=h_{\text {iso }}$. In Figure 3, the plane of the principal stresses $\left(\sigma_{1}, \sigma_{2}\right)$ for a damage state induced by a tension made in direction 1 is shown.

$$
\boldsymbol{D}=\left[\begin{array}{ccc}
D_{1} & 0 & 0 \\
0 & D_{2}=D_{1} / 2 & 0 \\
0 & 0 & D_{3}=D_{2}=D_{1} / 2
\end{array}\right]
$$

An induced anisotropy is obtained but a complete experimental study of yield surfaces of damage materials is necessary to conclude on the relevance or not of the coupling proposed between plasticity and damage.

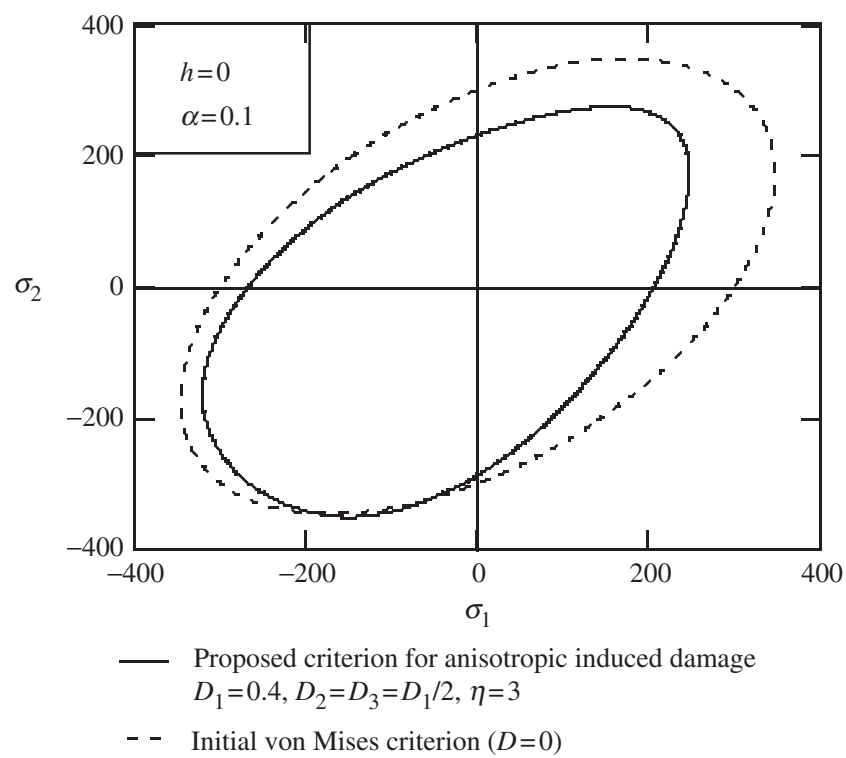

Figure 3. Convex yield surface with anisotropic damage due to tension $\left(\sigma_{y}+R=300 \mathrm{MPa}\right.$, $\mathbf{X}=0$ ). 


\section{DAMAGE EVOLUTION}

Finally, the energy function $\bar{Y}$ to be introduced in the anisotropic damage evolution law (14) is chosen as:

$$
\begin{aligned}
\bar{Y}= & \frac{1+v}{3 E}\left[\left(\boldsymbol{H}^{p} \boldsymbol{\sigma}_{+}^{D} \boldsymbol{H}^{p}\right)_{\mathrm{eq}}^{2}+h\left(\boldsymbol{H}^{n} \boldsymbol{\sigma}_{-}^{D} \boldsymbol{H}^{m}\right)_{\mathrm{eq}}^{2}\right] \\
& +\frac{3(1-2 v)}{2 E}\left[\frac{\left\langle\sigma_{H}\right\rangle_{+}^{2}}{\left(1-\eta D_{H}\right)^{2}}+h \frac{\left\langle\sigma_{H}\right\rangle_{-}^{2}}{\left(1-h \eta D_{H}\right)^{2}}\right]
\end{aligned}
$$

This models a damage growth slower in compression than in tension as for the same stress (in absolute value):

$$
\dot{D}_{\text {compression }} \approx\left(\frac{h+(2 / 9)(1-h)(1+v)}{1-(2 / 9)(1-h)(1+v)}\right)^{s} \dot{D}_{\text {tension }}
$$

or for $h=0$ :

$$
\dot{D}_{\text {compression }} \approx\left(\frac{2(1+v)}{7-2 v}\right)^{S} \dot{D}_{\text {tension }}<<\dot{D}_{\text {tension }}
$$

\section{DAMAGE GROWTH IN TENSION, COMPRESSION, SHEAR, AND PLANE TENSION}

The microdefects closure effect leads to an elasticity dissymmetric in tension and in compression. The interesting point concerning damage growth is that the proposed thermodynamics formulation which models this feature (through $h$ ) modifies the strain energy release rate in such a way that $\dot{D}$ in compression is much smaller than $\dot{D}$ in tension obtained for the same von Mises stress. The damage laws (12) and (14) also depend on the stress triaxiality, the higher the triaxiality the higher is the damage rate (Rice and Tracey, 1969).

The QU conditions challenge the triaxiality effect for damage growth but as illustrated in the next sections for different materials loaded in tension, compression and shear, their effect is complementary. The damage versus accumulated plastic strain curves are calculated and plotted next. These curves are not intrinsic characteristics of the material as they are loading-dependent. 


\section{Damage vs Accumulated Plastic Strain Curves}

Damage often occurs at saturated hardening $R=R_{\infty}=$ constant. The $D(p)$ curves are obtained then by performing the time integration of the damage evolutions laws (12) and (14). To simplify the calculations the yield surfaces with microdefects closure effect will be approximated by $\tilde{\sigma}_{\mathrm{eq}}-\sigma_{u} \approx 0$ with $\sigma_{u}=R_{\infty}+\sigma_{\mathrm{y}}$ the ultimate stress. Note that such a relationship stands exactly for the models without microdefects closure effect.

\section{TENSION}

Uniaxial tension corresponds to a state of stress

$$
\boldsymbol{\sigma}=\left[\begin{array}{lll}
\sigma & 0 & 0 \\
0 & 0 & 0 \\
0 & 0 & 0
\end{array}\right] \quad \sigma>0 \quad \sigma_{\mathrm{eq}}=\sigma \quad \sigma_{H}=\frac{\sigma}{3}
$$

The stress triaxiality $T_{X}=\sigma_{\mathrm{eq}} / \sigma_{H}$ is equal to $1 / 3$ which corresponds to a triaxiality function $R_{v}=1$.

- Isotropic damage with or without microdefects closure effect:

$$
D=\left(\frac{\sigma_{u}^{2}}{2 E S}\right)^{s}\left(p-p_{D}\right) \text { tension }
$$

- Anisotropic damage without microdefects closure effect: The damage state is represented by tensors $\boldsymbol{D}$ and $\boldsymbol{H}=(\mathbf{1}-\boldsymbol{D})^{-1 / 2}$

$$
\boldsymbol{D}=\left[\begin{array}{ccc}
D_{1} & 0 & 0 \\
0 & D_{1} / 2 & 0 \\
0 & 0 & D_{1} / 2
\end{array}\right] \quad \boldsymbol{H}=\left[\begin{array}{ccc}
\frac{1}{\sqrt{1-D_{1}}} & 0 & 0 \\
0 & \frac{1}{\sqrt{1-\left(D_{1} / 2\right)}} & 0 \\
0 & 0 & \frac{1}{\sqrt{1-\left(D_{1} / 2\right)}}
\end{array}\right]
$$

and the effective triaxiality function depends on the damage,

$$
\tilde{R}_{\nu U T}=\frac{2}{3}(1+v)+3(1-2 v)\left[\left(1-\frac{2 \eta}{3} D_{1}\right)\left(\frac{2}{1-D_{1}}+\frac{1}{1-\left(D_{1} / 2\right)}\right)\right]^{-2}
$$

The $D(p)$ curve is more easily calculated as a $p(D)$ relationship,

$$
p=p_{D}+\left(\frac{2 E S}{\sigma_{u}^{2}}\right)^{s} \int_{0}^{D} \tilde{R}_{\nu U T}^{-s} \mathrm{~d} D_{1} \quad \text { tension }
$$


- Anisotropic damage with microdefects closure effect: The damage state is still given by (63) with now:

$$
\begin{aligned}
\boldsymbol{H}^{p} & =\left[\begin{array}{ccc}
\frac{1}{\sqrt{1-D_{1}}} & 0 & 0 \\
0 & \frac{1}{\sqrt{1-\left(D_{1} / 2\right)}} & 0 \\
0 & 0 & \frac{1}{\sqrt{1-\left(D_{1} / 2\right)}}
\end{array}\right] \\
\boldsymbol{H}^{n} & =\left[\begin{array}{ccc}
\frac{1}{\sqrt{1-h D_{1}}} & 0 & 0 \\
0 & \frac{1}{\sqrt{1-\left(h D_{1} / 2\right)}} & 0 \\
0 & 0 & \frac{1}{\sqrt{1-\left(h D_{1} / 2\right)}}
\end{array}\right]
\end{aligned}
$$

For proportional loading the matrix $\boldsymbol{\sigma}^{D}$ and $\boldsymbol{H}^{p \text { or n }}$ commute and:

$$
\boldsymbol{\sigma}_{+}^{D}=\langle\boldsymbol{\sigma}\rangle_{+}=\left[\begin{array}{ccc}
\frac{2}{3} \sigma & 0 & 0 \\
0 & 0 & 0 \\
0 & 0 & 0
\end{array}\right] \quad \boldsymbol{\sigma}_{-}^{D}=\langle\boldsymbol{\sigma}\rangle_{-}=\left[\begin{array}{ccc}
0 & 0 & 0 \\
0 & -\frac{1}{3} \sigma & 0 \\
0 & 0 & -\frac{1}{3} \sigma
\end{array}\right]
$$

The expression for $\bar{Y}$ is then (Equation (58)):

$$
\bar{Y}=\frac{1+v}{3 E}\left[\frac{4 \sigma^{2}}{9\left(1-D_{1}\right)^{2}}+\frac{h \sigma^{2}}{9\left(1-\left(h D_{1} / 2\right)^{2}\right.}\right]+\frac{3(1-2 v)}{2 E} \frac{\sigma^{2}}{9\left(1-\frac{2}{3} \eta D_{1}\right)^{2}}
$$

or

$$
\begin{aligned}
\bar{Y} & =\frac{\sigma^{2}}{2 E} g_{\mathrm{YUT}}\left(D_{1}\right) \\
g_{\mathrm{YUT}}\left(D_{1}\right) & =\frac{1+v}{27 E}\left[\frac{8}{\left(1-D_{1}\right)^{2}}+\frac{2 h}{\left(1-\left(h D_{1} / 2\right)\right)^{2}}\right]+\frac{1-2 v}{3\left(1-(2 / 3) \eta D_{1}\right)^{2}}
\end{aligned}
$$


and the effective equivalent stress reads:

$$
\begin{aligned}
\tilde{\sigma}_{\mathrm{eq}} & =\sigma g_{\sigma \mathrm{UT}}\left(D_{1}\right) \\
g_{\sigma \mathrm{UT}}\left(D_{1}\right) & =\frac{1}{3} \sqrt{\frac{4}{\left(1-D_{1}\right)^{2}}+\frac{1}{\left(1-\left(h D_{1} / 2\right)\right)^{2}}+\frac{4}{\left(1-D_{1}\right)\left(1-\left(h D_{1} / 2\right)\right)^{2}}}
\end{aligned}
$$

Assuming finally $\tilde{\sigma}_{\mathrm{eq}} \approx \sigma_{u}$ gives:

$$
p=p_{D}+\left(\frac{2 E S}{\sigma_{u}^{2}}\right)^{s} \int_{0}^{D}\left[\frac{g_{\sigma \mathrm{UT}}^{2}\left(D_{1}\right)}{g_{\mathrm{YUT}}\left(D_{1}\right)}\right]^{s} \mathrm{~d} D_{1} \quad \text { tension }
$$

If the damage is measured for different values of the accumulated plastic strains, the previous closed form expressions allow to identify by curve fitting and for each model the damage threshold $p_{D}$ and the damage parameter $S$ for a known $s$. Only if tension experiments are available use the classical value $s=1$. If fatigue experiments are available use the Wöhler curve to identify $s$ (Lemaitre and Dufailly, 1987; Lemaitre and Demorat, 2005). If torsion and/or plane tension experiments are available use the expressions derived in the following paragraphs to identify simultaneously $S$ and $s$ from two $D(p)$ curves with different stress triaxialities.

\section{COMPRESSION}

Compression corresponds to a state of stress

$$
\boldsymbol{\sigma}=\left[\begin{array}{lll}
\sigma & 0 & 0 \\
0 & 0 & 0 \\
0 & 0 & 0
\end{array}\right] \quad \sigma<0 \quad \sigma_{\mathrm{eq}}=-\sigma \quad \sigma_{H}=\frac{\sigma}{3}
$$

with $T_{X}=\sigma_{\text {eq }} / \sigma_{H}=-1 / 3, R_{v}=1$. The models without microdefects closure effect give of course the same results than for tension.

- Isotropic damage without microdefects closure effect:

$$
D=\left(\frac{\sigma_{u}^{2}}{2 \mathrm{ES}}\right)^{s}\left(p-p_{D}\right) \quad \text { compression }
$$

- Isotropic damage with microdefects closure effect:

$$
D=\left(\frac{h_{\mathrm{iso}} \sigma_{u}^{2}}{2 \mathrm{ES}}\right)^{s}\left(p-p_{D}\right) \quad \text { compression }
$$


- Anisotropic damage without microdefects closure effect:

$$
p=p_{D}+\left(\frac{2 \mathrm{ES}}{\sigma_{u}^{2}}\right)^{s} \int_{0}^{D} \tilde{R}_{\nu \mathrm{UT}}^{-s} \mathrm{~d} D_{1} \quad \text { compression }
$$

- Anisotropic damage with microdefects closure effect:

$\boldsymbol{H}^{p}, \boldsymbol{H}^{n}$ are still given by Equation (66) and now:

$$
\boldsymbol{\sigma}_{+}^{D}=\left\langle\boldsymbol{\sigma}^{D}\right\rangle_{+}=\left[\begin{array}{ccc}
0 & 0 & 0 \\
0 & -\frac{1}{3} \sigma & 0 \\
0 & 0 & -\frac{1}{3} \sigma
\end{array}\right] \quad \boldsymbol{\sigma}_{-}^{D}=\left\langle\boldsymbol{\sigma}^{D}\right\rangle_{-}=\left[\begin{array}{ccc}
\frac{2}{3} \sigma & 0 & 0 \\
0 & 0 & 0 \\
0 & 0 & 0
\end{array}\right]
$$

The calculation is similar to the tensile case, it ends up to:

$$
p=p_{D}+\left(\frac{2 \mathrm{ES}}{\sigma_{u}^{2}}\right)^{S} \int_{0}^{D}\left[\frac{g_{\sigma C}^{2}\left(D_{1}\right)}{g_{Y C}\left(D_{1}\right)}\right]^{S} \mathrm{~d} D_{1} \quad \text { compression }
$$

with:

$$
\begin{aligned}
& g_{Y C}\left(D_{1}\right)=\frac{1+v}{27}\left[\frac{8 h}{\left(1-h D_{1}\right)^{2}}+\frac{2}{\left(1-\left(D_{1} / 2\right)\right)^{2}}\right]+\frac{h(1-2 v)}{3\left(1-(2 / 3) h \eta D_{1}\right)^{2}} \\
& g_{\sigma C}\left(D_{1}\right)=\frac{1}{3} \sqrt{\frac{4}{\left(1-h D_{1}\right)^{2}}+\frac{1}{\left(1-\left(D_{1} / 2\right)\right)^{2}}+\frac{4}{\left(1-h D_{1}\right)\left(1-\left(D_{1} / 2\right)\right)^{2}}}
\end{aligned}
$$

As already pointed out with the approximate expressions (41) and (60), there is a strong effect of the microdefects closure effect on the damage growth in compression (compared to the damage growth in tension for the same von Mises stress).

\section{SHEAR}

Shear corresponds to a state of stress

$$
\boldsymbol{\sigma}=\left[\begin{array}{lll}
0 & \tau & 0 \\
\tau & 0 & 0 \\
0 & 0 & 0
\end{array}\right] \quad \tau>0 \quad \sigma_{\mathrm{eq}}=\sqrt{3} \tau \quad \sigma_{H}=0
$$

with $T_{X}=\sigma_{\text {eq }} / \sigma_{H}=0, R_{v}=(2 / 3)(1+v)$. 
- Isotropic damage without microdefects closure effect:

$$
D=\left(\frac{(1+v) \sigma_{u}^{2}}{3 \mathrm{ES}}\right)^{s}\left(p-p_{D}\right) \text { shear }
$$

- Isotropic damage with microdefects closure effect: The positive and negative parts of $\boldsymbol{\sigma}=\boldsymbol{\sigma}^{D}$ in terms of principal values are:

$$
\langle\boldsymbol{\sigma}\rangle_{+}=\left[\begin{array}{ccc}
\frac{\tau}{2} & \frac{\tau}{2} & 0 \\
\frac{\tau}{2} & \frac{\tau}{2} & 0 \\
0 & 0 & 0
\end{array}\right] \quad\langle\boldsymbol{\sigma}\rangle_{-}=\left[\begin{array}{ccc}
-\frac{\tau}{2} & \frac{\tau}{2} & 0 \\
\frac{\tau}{2} & -\frac{\tau}{2} & 0 \\
0 & 0 & 0
\end{array}\right]
$$

and then:

$$
\begin{gathered}
Y=\frac{1+v}{E} \tau g_{Y S}^{0}(D) \\
\mathrm{g}_{Y S}^{0}(D)=\frac{1}{2}\left[\frac{1}{(1-D)^{2}}+\frac{h}{(1-h D)^{2}}\right] \\
\tilde{\sigma}_{\mathrm{eq}}=\sqrt{3} \tau g_{\tau S}^{0}(D) \\
g_{\tau S}^{0}(D)=\sqrt{\frac{5}{18(1-D)^{2}}+\frac{5}{18(1-h D)^{2}}+\frac{4}{9(1-D)(1-h D)}}
\end{gathered}
$$

and

$$
p=p_{D}+\left(\frac{3 E S}{(1+v) \sigma_{u}^{2}}\right)^{s} \int_{0}^{D}\left[\frac{g_{\tau S}^{0}{ }^{2}(D)}{g_{Y S}^{0}(D)}\right]^{s} \mathrm{~d} D \quad \text { shear }
$$

- Anisotropic damage without microdefects closure effect: The damage is isotropic in the shear plane:

$$
\boldsymbol{D}=\left[\begin{array}{lll}
D & 0 & 0 \\
0 & D & 0 \\
0 & 0 & 0
\end{array}\right]
$$

with then

$$
\bar{Y}=\frac{(1+v) \sigma_{u}^{2}}{3 E}
$$


and for the isotropic case,

$$
D=\left(\frac{(1+v) \sigma_{u}^{2}}{3 E S}\right)^{s}\left(p-p_{D}\right) \quad \text { shear }
$$

- Anisotropic damage with microdefects closure effect: The damage state is still given by Equation (86) with now:

$$
\begin{aligned}
\bar{Y} & =\frac{1+v}{3 E} \tau^{2} g_{\mathrm{YS}}(D) \\
g_{\mathrm{YS}}(D) & =\frac{1}{2}\left[\frac{1}{(1-D)^{2}}+\frac{h}{(1-h D)^{2}}\right] \\
\tilde{\sigma}_{\mathrm{eq}} & =\sqrt{3} \tau g_{\tau S}(D) \\
g_{\tau S}(D) & =\sqrt{\frac{1}{3(1-D)^{2}}+\frac{1}{3(1-h D)^{2}}+\frac{z 1}{3(1-D)(1-h D)}}
\end{aligned}
$$

and

$$
p=p_{D}+\left(\frac{3 \mathrm{ES}}{(1+v) \sigma_{u}^{2}}\right)^{s} \int_{0}^{D}\left[\frac{g_{\tau S}^{2}(D)}{g_{Y S}(D)}\right]^{S} \mathrm{~d} D \text { shear }
$$

First, the damage laws studied give rise to damage growth in shear. Then, to take into account or not the microdefects closure effect in shear gives a different damage growth (lower when the unilateral effect is activated through $h_{\text {iso }}<1$ or $h<1$ ). This can be explained by recalling that shear is in fact a biaxial tension-compression state of stress if written in the framework built with the principal directions of $\boldsymbol{\sigma}$. The microcracks in the tension direction mainly open when the microcracks in the compression direction mainly close.

\section{PLANE TENSION}

In case of large plane specimens a (plane) tension loading ensures a plane strain state in the middle of each sheet. If $\vec{x}_{1}$ is the direction of tension, $\vec{x}_{2}$ the transverse direction and $\vec{x}_{3}$ the direction in the thickness, the strain $\epsilon_{22}$ vanishes due to the plane strain condition. Furthermore, if during loading elastic strains are neglected then $\epsilon_{22}^{p} \approx 0$ and

$$
\boldsymbol{\epsilon}^{p}=\left[\begin{array}{ccc}
\epsilon_{11}^{p} & 0 & 0 \\
0 & 0 & 0 \\
0 & 0 & -\epsilon_{11}^{p}
\end{array}\right]
$$


The states of stress is then:

$$
\boldsymbol{\sigma}=\left[\begin{array}{ccc}
\sigma & 0 & 0 \\
0 & \frac{\sigma}{2} & 0 \\
0 & 0 & 0
\end{array}\right]
$$

with $T_{X}=\sigma_{\text {eq }} / \sigma_{H}=1 / \sqrt{3}, R_{v}=R_{v P T}=(2 / 3)(1+v)+(1-2 v)$.

- Isotropic damage without or with microdefects closure effect:

$$
D=\left(\frac{\sigma_{u}^{2} R_{v P T}}{2 E S}\right)^{s}\left(p-p_{D}\right) \quad \text { plane tension }
$$

- Anisotropic damage without microdefects closure effect: The damage law (14) gives $D_{3}=D_{1}, D_{2}=0$. The state of damage is then:

$$
\boldsymbol{D}=\left[\begin{array}{ccc}
D_{1} & 0 & 0 \\
0 & 0 & 0 \\
0 & 0 & D_{1}
\end{array}\right]
$$

The effective stress triaxiality depends upon damage,

$$
\tilde{R}_{v P T}\left(D_{1}\right)=\frac{2}{3}(1+v)+(1-2 v)\left(\frac{1-D_{1}}{1-(2 \eta / 3) D_{1}}\right)^{2} \quad \text { plane tension }
$$

and the $D(p)$ curve is given by:

$$
p=p_{D}+\left(\frac{2 E S}{\sigma_{u}^{2}}\right)^{s} \int_{0}^{D_{1}} \tilde{R}_{v P T}^{-s}\left(D_{1}\right) \mathrm{d} D_{1}
$$

- Anisotropic damage with microdefects closure effect: The damage state is still given by Equation (94) and

$$
p=p_{D}+\left(\frac{2 E S}{\sigma_{u}^{2}}\right)^{S} \int_{0}^{D}\left[\frac{g_{\sigma P T}^{2}\left(D_{1}\right)}{g_{Y P T}\left(D_{1}\right)}\right]^{S} \mathrm{~d} D \text { plane tension }
$$

with:

$$
\begin{aligned}
\bar{Y}\left(D_{1}\right) & =\frac{\sigma^{2}}{2 E} g_{\mathrm{YPT}}\left(D_{1}\right) \\
\tilde{\sigma}_{\mathrm{eq}}\left(D_{1}\right) & =\sigma g_{\sigma \mathrm{PT}}\left(D_{1}\right)
\end{aligned}
$$


and:

$$
\begin{aligned}
g_{\mathrm{YPT}}\left(D_{1}\right) & =\frac{1+v}{3}\left[\frac{1}{2\left(1-D_{1}\right)^{2}}+\frac{h}{2\left(1-h D_{1}\right)^{2}}\right]+\frac{3(1-2 v)}{4\left(1-(2 / 3) \eta D_{1}\right)^{2}} \\
g_{\sigma \mathrm{PT}}\left(D_{1}\right) & =\sqrt{\frac{1}{4\left(1-D_{1}\right)^{2}}+\frac{1}{4\left(1-h D_{1}\right)^{2}}-\frac{1}{4\left(1-D_{1}\right)\left(1-h D_{1}\right)}}
\end{aligned}
$$

Plane tension tests may complete the experimental database for the identification of the damage exponent $s$.

To sum up, these closed form expressions allow one to plot easily the $D(p)$ curves for the different loading. Depending on the available experimental data, the damage parameters can be identified once the hardening parameters are known either:

- on uniaxial tension results only. Take then $s=1$ or identify $s$ from fatigue results.

- on uniaxial tension and torsion results. This gives explicitly the value of $s$.

- on uniaxial tension and plane tension results if the testing specimens are large sheets. This gives explicitly the value of $s$.

Take then $h_{\text {iso }}=0.2$ or $h=0$ to use the models with microdefects closure effect.

\section{Stainless Steels 2 1/4 Cr Mo and 4 1/2 Cr Mo}

The testing specimens are plain bars of diameter $6 \mathrm{~mm}$. Tension, torsion and tension followed by torsion experiments have been performed by Fontaine (1985). Because of the massive specimens, the loading was applied up to failure with no instabilities in torsion: six or seven full rotations were needed to break the specimens. The drawback of using plain bars is that damage is not uniform in torsion and that structure calculations in finite strains are needed to represent the experiments. Such calculations have not been performed, nevertheless some interesting features were qualitatively pointed out:

- Failure occurs in torsion after several rotations which corresponds to an amount of accumulated plastic strain much larger than in tension.

- The average critical damage measured in torsion is of the same order of magnitude than the critical damage $D_{\mathrm{c}}$ measured in tension.

A qualitative diagram $D(p)$ which summarizes these features may then be drawn (Figure 4). Quantitative measurements and calculations are given next for two different materials. 


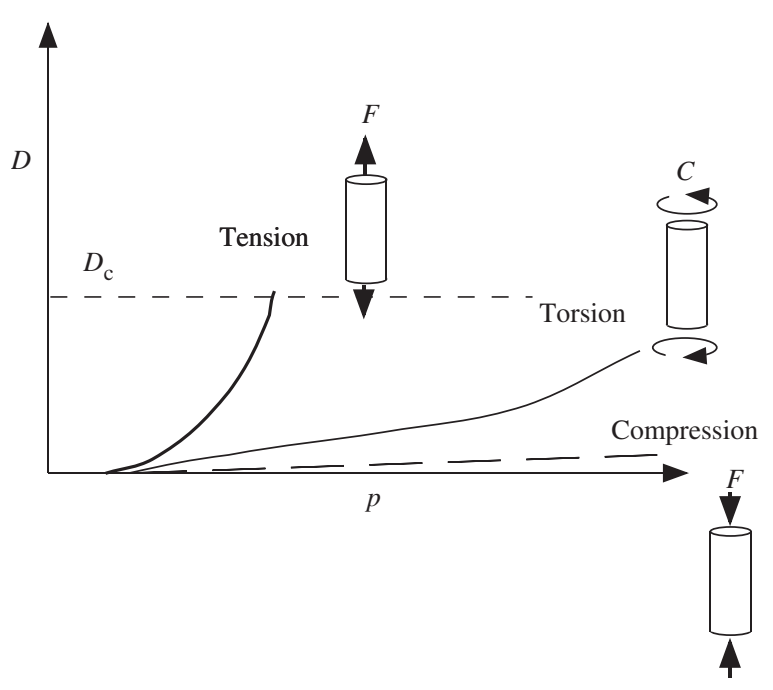

Figure 4. Qualitative $D(p)$ curves.

\section{FCD 400 Spheroidized Cast Iron}

The testing specimens are tubes of $1 \mathrm{~mm}$ thickness and $17 \mathrm{~mm}$ diameter. The tubes are too thin to be loaded up to failure with no instabilities in torsion but they induce uniform states of stress, strain, and damage. Damage is then directly measured from the global stiffness of the specimen as repeated unloadings were performed. Yakawaka and Murakami's (1997) results of loss in Young's modulus versus the axial strain and of loss in shear modulus versus shear strain are used.

von Mises criterion with an exponential isotropic hardening is used. Damage occurs here before the hardening saturation and a hardening evolution $R=R_{\infty}(1-\exp (-b p))$ is assumed. An average expression of the hardening law represents both tension and torsion experiments (Figure 5). The damage is calculated by keeping $\sigma_{u}=R(p)+\sigma_{\mathrm{y}}$ in an integral over $p$, therefore by replacing in the previous $p(D)$ closed form expressions the term $\sigma_{u}^{2}\left(p-p_{D}\right)$ by $\int_{p_{D}}^{p}\left(R(p)+\sigma_{\mathrm{y}}\right)^{2} \mathrm{~d} p$.

The material parameters of the damage law are identified from the measured loss of stiffness in tension with the usual values $s=1, h_{\text {iso }}=0.2$, and with a zero damage threshold $p_{D}$. The final sets of the damage parameters for the FCD 400 cast iron are:

- $E=200000 \mathrm{MPa}, v=0.3$ for elasticity,

- $\sigma_{\mathrm{y}}=300 \mathrm{MPa}$ as yield stress,

- $R_{\infty}=170 \mathrm{MPa}, b=22$ for hardening, 


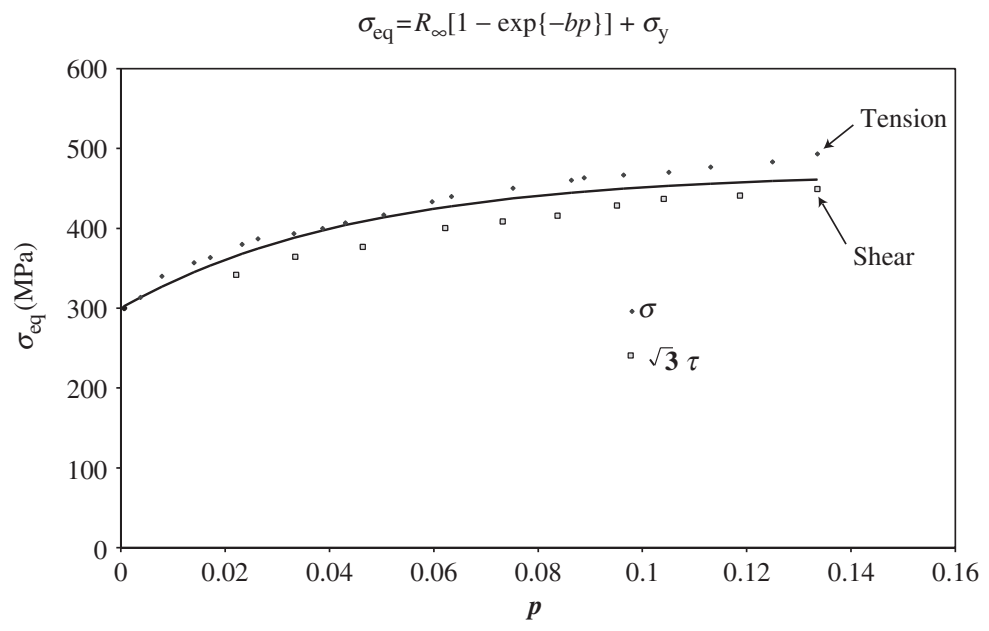

Figure 5. Identification of the hardening parameters.

- $S=0.66 \mathrm{MPa}, h_{\text {iso }}=1$ for isotropic damage without microdefect closure effect,

- $S=0.66 \mathrm{MPa}, h_{\text {iso }}=0.2$ for isotropic damage with microdefect closure effect,

- $S=0.45 \mathrm{MPa}, \eta=2.15, h=1$, for anisotropic damage without microdefects closure effect,

- $S=0.45 \mathrm{MPa}, \eta=2.15, h=0.2$, for anisotropic damage with microdefects closure effect.

The corresponding $D(p)$ curves of Figure 6 show an excellent agreement in shear when the microdefects closure effect is taken into account (QU conditions). Again, this may be partly explained by recalling that shear is in fact a biaxial state of tension and compression in two orthogonal (principal) directions.

\section{SOLDUR 355 Dispersoid Steel}

Consider finally Sollac SOLDUR 355 steel which, due to the forming process, comes in large plates appropriate neither for torsion nor for compression experiments (Lemaitre et al., 2000). The plates are loaded, plastified, and damaged in both tension and plane tension cases with different stress triaxialities $T_{X}=\sigma_{H} / \sigma_{\text {eq }}$. In tension $T_{X}=1 / 3$ when in plane tension due to the plane strain condition with respect to the transverse direction $T_{X} \approx 0.58$.

In order to measure the damage (its value, its anisotropy) small tensile specimens have been cut in the sheets loaded in tension and in plane tension 


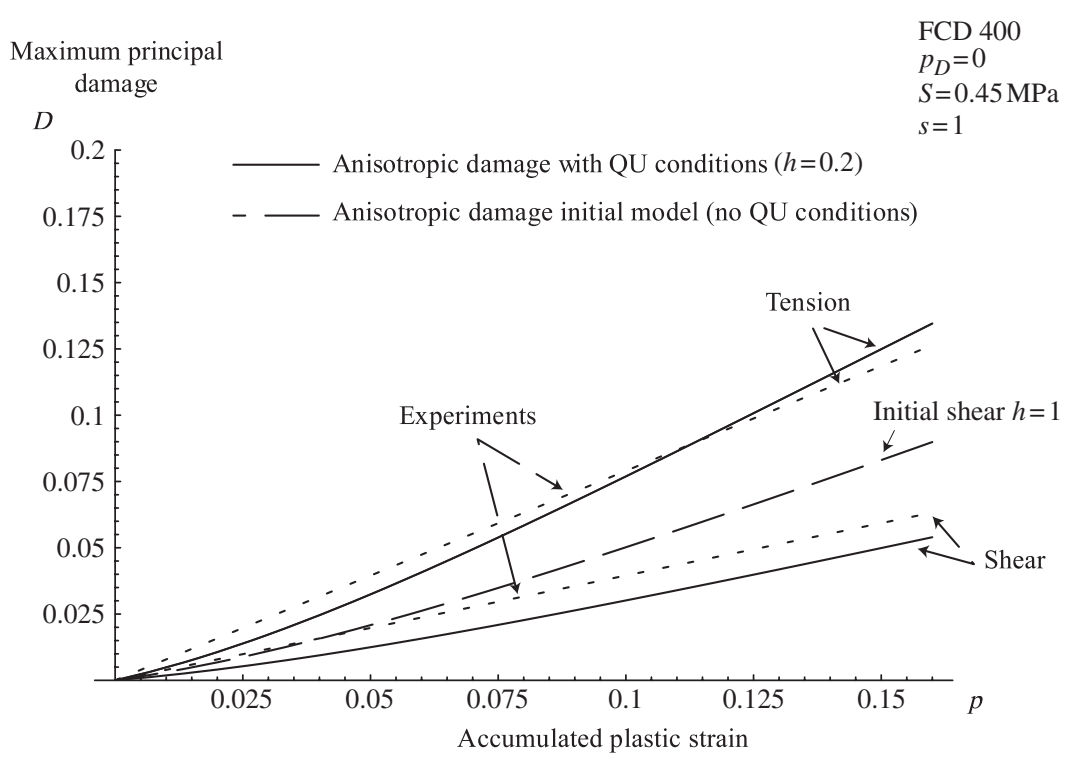

Figure 6. $D(p)$ curves for $F C D$ cast iron.

(Figure 7) and tested elastically. The full experimental procedure leads to the validation of the anisotropic damage law (Lemaitre et al., 2000). The feature $D_{2}=D_{3}=D_{1} / 2$ in tension is recovered with $\eta=3$ for the anisotropic damage model without microdefect closure effect, with $\eta=2.15$ for anisotropic damage with microdefect closure effect.

The set of material parameters for anisotropic damage are identified on both uniaxial tension and plane tension:

- $E=200000 \mathrm{MPa}, v=0.3$ for elasticity,

- $\sigma_{\mathrm{y}}=300 \mathrm{MPa}$ as yield stress,

- $R_{\infty}=174 \mathrm{MPa}$ for saturated hardening,

- $p_{D}=0.025$ as damage threshold,

- $S=0.527 \mathrm{MPa}, s=3.8, \eta=3, h_{\text {iso }}=0.2, D_{c}=0.2$ for isotropic damage with microdefects closure effect,

- $S=0.527 \mathrm{MPa}, s=3.8, \eta=3, h_{\text {iso }}=1, D_{c}=0.2$ for isotropic damage without microdefects closure effect,

- $S=0.57 \mathrm{MPa}, s=4, \eta=3, h=1, D_{c}=0.2$ for anisotropic damage without microdefects closure effect,

- $S=0.34 \mathrm{MPa}, s=2.5, \eta=2.1, h=0, D_{c}=0.2$ for anisotropic damage with microdefects closure effect.

The $D(p)$ curves are plotted in Figure 8 in case of anisotropic damage without or with microdefects closure effect. The plane tension and the 


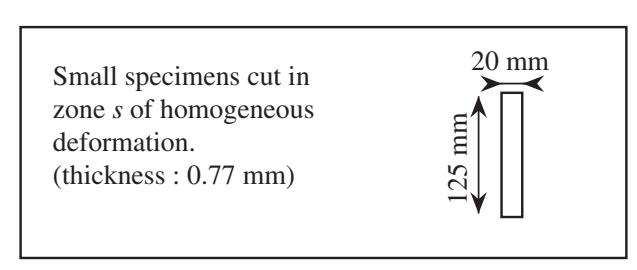

Uniaxial tension large specimens

Plane tension large specimens
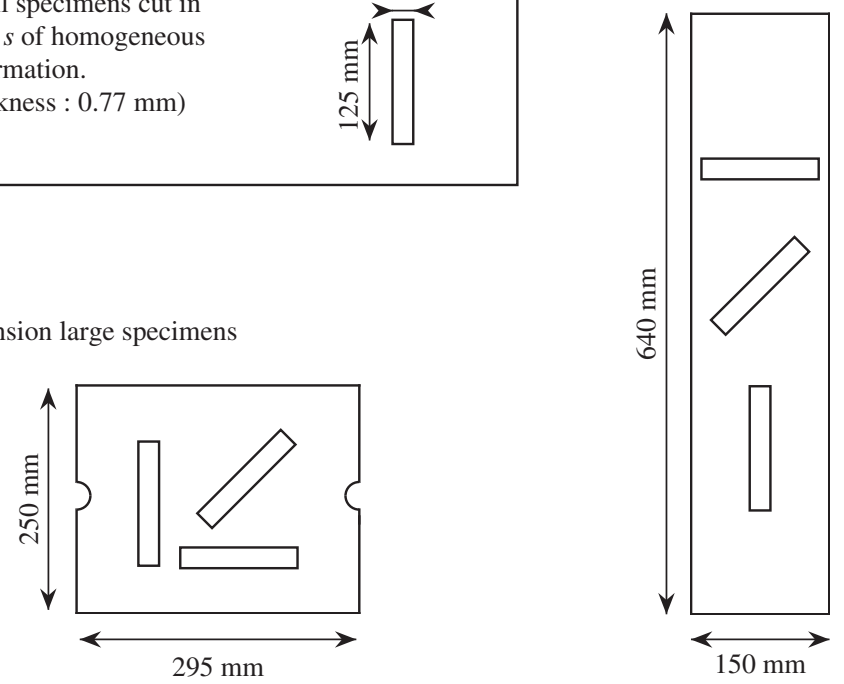

Figure 7. Small specimens cut in large specimens.

Anisotropic damage laws with/without unilateral effect

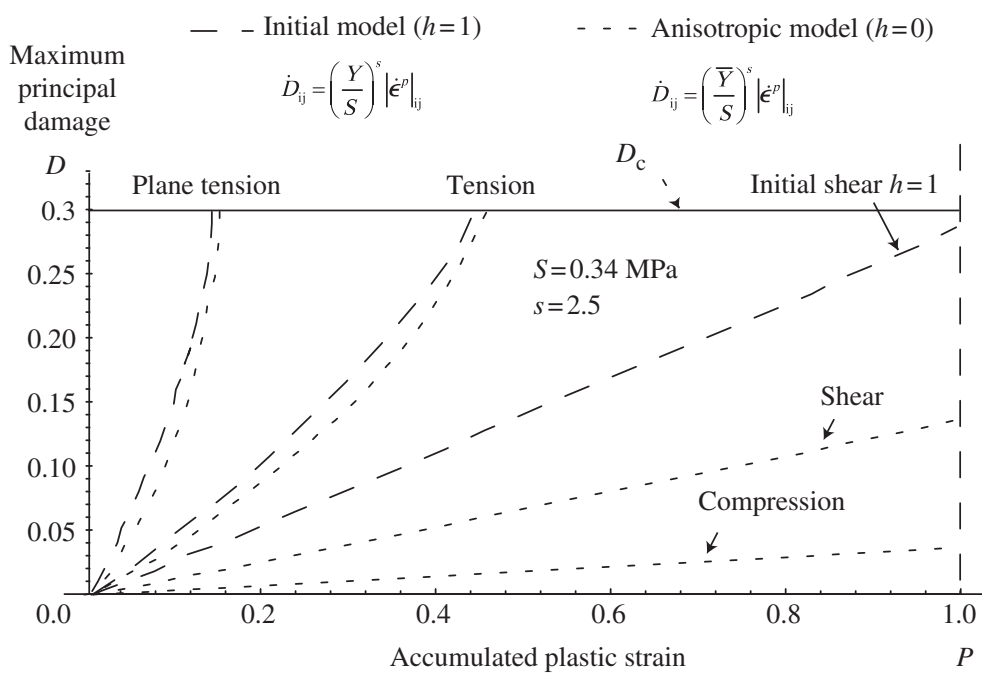

Figure 8. $D(p)$ curves for SOLDUR 355 dispersoid steel. 
uniaxial tension curves match because they are the basis for the damage parameters identification. The shear and compression curves are predictions, fully realistic if compared to the results obtained for the $2 \frac{1}{4} \mathrm{Cr} \mathrm{Mo}, 4 \frac{1}{2}$ Cr Mo steels, and FCD 400 cast iron. Recall that the compression case without microdefects closure effect is identical to the tension case. This shows that the anisotropic damage law represents well the damage anisotropy and the damage growth for different loadings, different in triaxiality but also in sign.

\section{CONCLUSION}

To conclude, it is possible to represent with the same model both triaxiality effect and microdefects closure effect on damage growth. The damage evolution laws remain the same as for the case without unilateral conditions (but with an adequate definition of $Y$ and $\bar{Y}$ ),

$$
\begin{aligned}
& \dot{D}=\left(\frac{Y}{S}\right)^{s} \dot{p} \quad \text { isotropic damage } \\
& \dot{D}=\left(\frac{Y}{S}\right)^{s}\left|\dot{\epsilon}^{p}\right| \text { anisotropic damage }
\end{aligned}
$$

laws which also apply in fatigue, creep and creep-fatigue (Desmorat, 2002; Lemaitre and Demorat, 2005).

The damage versus accumulated plastic strain curves have been calculated in tension, compression, shear and plane tension. Closed form expressions $D=D(p)$ or $p=p(D)$ have been obtained and can then be used to identify the damage parameters from different monotonic experiments.

Concerning the yield criterion for damaged materials, it has been pointed out that for models with microdefects closure effect to replace the stress $\sigma$ by the effective stress $\tilde{\boldsymbol{\sigma}}$ leads to nonconvex yield surfaces. A theoretically correct coupling between plasticity and damage has been formulated.

\section{REFERENCES}

Cordebois, J.P. and Sidoroff, F. (1982). Endommagement anisotrope en élasticité et plasticité, J.M.T.A., Numéro spécial, pp. 45-60.

Chaboche, J.L. (1978). Description Phenomenologique de la Viscoplasticite Cyclic avec Endommagement. Doctorat d'Etat, Universite Pierre et Marie Curie, Paris 6, Paris (Juin).

Chaboche, J.L. (1993). Development of Continuum Damage Mechanics for Elastic Solids Sustaining Anisotropic and Unilateral Damage, Int. J. Damage Mech., 2: 311-329. 
Chow, C.L. and Wang, J. (1987). An Anisotropic Theory for Continuum Damage Mechanics, Int. J. Fract., 33: 3-16.

Desmorat, B. (2002). Optimisation de structures composites, PhD Thesis of Ecole Normale Suprieure de Cachan.

Desmorat, R. (2000a). Strain Localization and Unilateral Conditions for Anisotropic Induced Damage Model, In: Benallal, A. (ed.), Symposium on Continuous Damage and Fracture, pp. 71-79, Elsevier, 23-27 October 2000, Cachan, France.

Desmorat, R. (2000b). Modélisation et estimation rapide de la plasticité et de l'endommagement, Mémoire d'Habilitation à Diriger des Recherches, Université Paris 6.

Desmorat, R. (2002). Fast Determination of Localized Plasticity and Damage by Energetic Methods, Int. J. of Solids and Structures, 39: 3289-3310.

Fontaine, J.F. (1985). PhD Thesis LMT Cachan, Université Paris 6.

Halm, D. and Dragon, A. (1998). An Anisotropic Model of Damage and Frictional Sliding for Brittle Materials, Env. J. Mechanics A/Solids, 17: 439-460.

Kachanov, L.M. (1958). Time of Rupture Process Under Creep Conditions, Izv. Akad. NAUK. SSR - Otd. Nauk., 8: 26-31.

Krajcinovic, D. (1985). Continuous Damage Mechanics Revisited: Basic Concepts and Definitions, J; Appl. Mech., 52: 829-834.

Ladevèze, P. (1983). On an Anisotropic Damage Theory, In: Boehler, J.P. (ed), Proc. CNRS Int. Coll. 351 Villars-de-Lans, Failure Criteria of Structured Media, pp. 355-363. (Also Internal report 34 of LMT Cachan).

Ladevèze, P. and Lemaitre, J. (1984). Damage Effective Stress in Quasi Unilateral Conditions, In: Proceedings of the 16th International Congress of Theoretical and Applied Mechanics, Lyngby, Denmark.

Ladevèze, P. (1995). Modeling and Simulation of the Mechanical Behavior of CMCs, In: Evans A.G. and Naslain R. (eds), High Temperature Ceramic-Matrix Composite, Ceramic Transaction, 57: 53-64.

Leckie, F.A. and Onat, E.T. (1981). Tensorial Nature of Damage Measuring Internal Variables, In: Hult J. and Lemaitre J. (eds), Physical Non-linearities in Structural Analysis, pp. 140-155, Springer, Berlin.

Lemaitre, J. (1971). Evaluation of Dissipation, Damage in Metals Submitted to Dynamic Loading, In: Proc. Ist International Conference on Mechanical Behavior of Materials.

Lemaitre, J. and Chaboche, J.L. (1985). Mécanique des Matériaux Solides, Dunod, Paris. Mechanics of Solids Materials, Cambridge University Press, Cambridge. English Translation 1990.

Lemaitre, J. and Dufailly, J. (1987). Damage Measurement, Eng. Fract. Mech., 28(5): 6643-6661.

Lemaitre, J. (1992). A Course on Damage Mechanics, Springer Verlag, Berlin.

Lemaitre, J., Demorat R. and Sauzay, M. (2000). Anisotropic Damage Law of Evolution, Eur. J. Mech. A/Solids, 19: 513-524.

Lemaitre J. and Demorat, R. (2001). Isotropic and Anisotropic Damage Law Evolution, In: Lemaitre, J. (ed.), Handbook of Materials Behavior Models, Academic Press, Chapter Continuous Damage, Section 6.14, pp. 513-524.

Lemaitre J. and Demorat R. (2005). Engineering Damage Mechanics: Ductile, Creep, Fatigue and Brittle Failures, Springer, Berlin.

Liénard, C. (1989). Plasticité couplée à l'endommagement en conditions quasi-unilatérales pour la prévision de l'amorçage de fissures, PhD thesis LMT Cachan, Universit Paris 6.

Murakami, S. (1988). Mechanical Modelling of Material Damage, J. App. Mech., 55: 280-286.

Rabotnov, Y.N. (1968). Creep Rupture, Proc. XII Int. Congress Appl. Mech. 
Rice J.R. and Tracey, D.M. (1969). On the Enlargement of Voids in Triaxial Stress Fields, J. Mech. Phys. Solids, 17: 201-217.

Sermage, J.-P., Lemaitre, J. and Desmorat, R. (2000). Multiaxial Creep-fatigue under Anisothermal Conditions, Fatigue Fract. Engng. Mater. Struct., 23: 241-252.

Yayakawa, K. and Murakami, S. (1997). Thermodynamical Modelling of Elastic-plastic Damage and Experimental Validation of Damage Potential, Int. J. Damage Mech., 6: $333-363$. 